\title{
Allostatic load and heart rate variability as health risk indicators.
}

\author{
Margaretha Viljoen ${ }^{1}$, Nicolaas Claassen ${ }^{2}$
}

1. Department of Psychiatry, School of Medicine, Faculty of Health Sciences, University of Pretoria, South Africa 2. School of Health Systems and Public Health, Faculty of Health Sciences, University of Pretoria, South Africa

\begin{abstract}
Background: Uncertainty often exists about the comparability of results obtained by different health risk indicator systems. Objectives: To compare two health risk indicator systems, i.e, allostatic load and heart rate variability (HRV). Additionally, to investigate the feasibility of inclusion of HRV indicators into allostatic load assessments and which HRV indicators are best to introduce.

Methods: Allostatic loads were calculated based on blood pressure, waist-to-hip ratio, BMI, cholesterol, HDL-C, LDL-C, CRP, albumin, glycosylated haemoglobin, blood glucose and cortisol excretion. Allostatic load scores were compared to HRV results obtained by frequency domain, time domain and Poincaré analyses.

Results: Negative correlations were found between allostatic loads and total HRV, for all periods and all HRV analytical techniques $(\mathrm{r}=-0.67, \mathrm{p}=0.0001$ to $\mathrm{r}=-0.435, \mathrm{p}=0.035)$, and between allostatic loads and vagal measures of HRV for supine $(\mathrm{r}=-0.592$, $\mathrm{p}=0.001$ to $\mathrm{r}=-0.584, \mathrm{p}=0.001)$ and the first 5 minutes standing $(\mathrm{r}=-0.443, \mathrm{p}=0.021$ to $\mathrm{r}=-0.407, \mathrm{p}=0.035)$, with all HRV techniques. Heart rate responses declined with increases in allostatic loads.

Conclusion: HRV and allostatic load scores give comparable results as health risk indicators. Baseline total HRV and vagal, rather than sympathetic, measures of HRV should be introduced into allostatic load assessments. Results are in line with the concept of vagal tone as a regulator of allostatic systems. Inclusion of heart rate responses to orthostatic stress, into allostatic load assessments, warrants further investigation.
\end{abstract}

Keywords: Psychosocial stress; allostatic load; heart rate variability.

DOI: https://dx.doi.org/10.4314/ahs.v17i2.17

Cite as: Viljoen M, Claassen N. Allostatic load and heart rate variability as health risk indicators. Afri Health Sci. 2017;17(2): 428-435. bttps:/ / dx.doi.org/10.4314/abs.v17i2.17

\section{Introduction}

Various tools are available to assess the wellness of populations working or living under conditions where psychosocial stressors may lead to a decline in physiological or psychological wellness. All of these tests or health risk indicator systems have limitations and virtually all have their own methodological challenges. Methodological heterogeneity between investigating laboratories may be a confounding factor in comparing results obtained by the same health risk indicator system ${ }^{1}$. The problem is further exacerbated when comparing the health status of populations assessed by different tests. Allostatic load
Corresponding author:
Margaretha Viljoen,
36 Buffels Road, Rietondale,
Pretoria, 0084, South Africa
Tel: +27 12329 3090;
Email: mviljoen@webafrica.org.za

and heart rate variability (HRV) are examples of assessments frequently used as health risk indicators. While both relate to activities of the main stress axes, no overlap exists between their respective analytical methodologies. It is of practical interest to know to what degree allostatic load scores correspond to information obtained by HRV determination. HRV indicators have on occasion been introduced as indices of allostatic load. It is essential that consensus be reached as to which indicators are best suited for inclusion.

Allostatic load is a multisystem approach to the assessment of physical health status. Allostatic load is described as the extent to which chronic, or a high frequency of intermittent stress, i.e, chronic or a high frequency of activation of the neuroendocrine stress responses, can lead to physiological wear and tear ${ }^{2}$. Initial calculation of allostatic load was done by summing across indices of subjects' status in terms of ten physiological variables said to reflect stressor-induced activation of the two main stress axes, i.e, the hypothalamo-pituitary-adreno- 
cortical axis (HPA-axis) and the autonomic nervous system $(\mathrm{ANS})^{2,3}$. The original ten variables comprised serum dihydroepiandrosterone sulphate, overnight urinary cortisol excretion, noradrenalin and adrenalin excretion, systolic and diastolic blood pressure, waist-to-hip ratio, serum high density lipoprotein cholesterol (HDL-C), total cholesterol and glycosylated haemoglobin ${ }^{2,3}$. Allostatic load was then calculated as the sum of the number of variables for which a subject was rated in the highest-risk quartile for that specific variable, with higher allostatic load scores indicating a greater health risk. However, various laboratories have since used their own adaptations of the method ${ }^{1}$. The main deviations from the original set of variables include the exclusion of noradrenalin and adrenalin and the inclusion of a pro-inflammatory marker such as neopterin or C-reactive protein.

Analysis of HRV, i.e, analysis of the fluctuation of heart period over time, is widely used as a measure of ANS status and flexibility ${ }^{4}$. Furthermore, HRV is considered to be a marker of psychological and physical well-being and as a predictor of morbidity and mortality ${ }^{4,5}$. HRV is reduced in stress and said to be of prognostic value for several chronic diseases ${ }^{4,5}$. However, HRV variances are not only linked to ANS-related disturbances, but also to that of the HPA-axis ${ }^{6}$. A small number of studies have introduced HRV as part of their allostatic load assessments - ostensibly as replacement for noradrenaline and adrenalin. However, consensus as to which HRV techniques and indicators to use has not yet been attempted.

The primary aim of this study was to test correlations between results obtained by allostatic load and that obtained by HRV. Allostatic load scores were compared to HRV results obtained by frequency domain, time domain and Poincaré analyses. A second aim was to investigate whether it is meaningful to include selected HRV indicators as indices of allostatic load, and if so, which indicators.

\section{Methods}

Allostatic loads and HRV were assessed in 27 full time employed male subjects.. Inclusion criteria was that all subjects were actively involved in armed protection, employed by the same company and had passed the routine assessment by the company's medical consultant. Subjects on any medication known to influence HRV were excluded from the study. Recruitment by a power point-illustrat- ed talk on the aims and procedures was performed in the company staff room. All subjects (28 males) employed in the relevant capacity volunteered. However, one potential participant had to be turned down on grounds of medical history and medication. Recruitment was followed by collecting information on medical histories, medication, working hours and anthropometric assessments. Clearance was obtained from the Ethics Committee, Faculty of Health Sciences, University of Pretoria and all volunteers signed informed consent prior to enrolment in the study.

Allostatic load was determined and calculated by the method of Seeman, et at ${ }^{13}$ with an adaptation in the parameters assessed. The following parameters were measured for calculation of allostatic load: systolic and diastolic blood pressure, waist-to-hip ratio, body mass index (BMI), serum high density lipoprotein cholesterol (HDL-C), low density lipoprotein cholesterol (LDL-C), total cholesterol, aldosterone, albumin, C-reactive protein (CRP), fasting blood glucose, glycosylated haemoglobin (\%HbA1c), and over-night (12 hours) urinary cortisol excretion. Blood for the biochemical analyses was collected between 08:00 and 09:00 at the laboratory where the biochemical analyses were performed. The allostatic load score for each subject was calculated by summing the number of parameters for which the value fell into the highest risk quartile (the top quartile for all parameters, except for HDL-cholesterol for which the lowest quartile represents the highest risk). The score based on the 13 parameters used could thus have varied between 0 and 13 with higher scores indicating higher health risk. Criteria for highest risk quartiles for the biochemical parameters were derived from the normal ranges of the clinical laboratory where the blood was analysed and that for blood pressure, BMI and waist-to-hip ratios were based on international accepted values. BMI was calculated as the weight in kilograms divided by the square of the individual's height in meters $\left(\mathrm{kg} / \mathrm{m}^{2}\right)$. Waist-to-hip ratio (waist measurement divided by hip measurement) was determined, at the end of a normal expiration with the individual standing with arms at the side. Waist was measured at the smallest circumference of the natural waist and hip at the widest circumference of the hip.

Tachogram recordings for the calculation of HRV were done over a continuous period analysed in the follow- 
ing phases: a 5 minute stabilization period in the supine position (these were discarded), 5 minute in the supine position for the baseline recordings ( $\mathrm{Ph} 0)$, followed by getting up into the standing position and remain standing for 5 minute (Ph1), and two 5 minute periods stationary in the standing position (Ph2 and Ph3). Standing up from supine (Ph0-Ph1) was used to test the response to orthostatic stress. The Actiheart chest-worn heart rate monitor (CamNtech Ltd, Cambridge, UK) was used to record inter-beat-intervals (IBI) by digitising the ECG signal from the R-R interval with a $1 \mathrm{~ms}$ resolution. IBI data series were stored in a Polar Precision Performance Software template file to enable error correction by means of Polar Precision Performance Software, version 4.03.040 (Polar Electro Oy, Kempele, Finland). HRV analysis was carried out using the advanced HRV Analysis 1.1 software for windows (Biomedical Signal Analysis Group, University of Kuopio, Finland).

HRV data was analysed by frequency domain analysis based on Fast Fourier Transformations (FFT), by frequency domain analysis based on autoregression (AR), by time-domain analysis and by Poincaré analysis. Analyses were performed in line with Task Force recommendations ${ }^{4}$. Before FFT was employed all imported data was detrended, using the smoothness priors method $^{7}$ and then interpolated at a sampling rate of $4 \mathrm{~Hz}$.

Frequency-domain analysis informs on the relative power of underlying intrinsic rhythms involved in the regulation of heart rate (HR). The high frequency (HF) band $(0.15-0.4 \mathrm{~Hz})$ represents predominantly parasympathetic (vagal) activity, while the low frequency (LF) band (0.04 $0.15 \mathrm{~Hz})$ is said to represent sympathetic activity, but with a parasympathetic component ${ }^{4}$. However, marked disagreement exists about the validity of LF as a reflection of sympathetic activity ${ }^{8}$. In view of that, and the wealth of evidence for an association of wellness with total HRV and with vagal-associated variability, the focus was on total HRV and vagal measures of HRV.

Time-domain measures, calculated from the error-corrected R-R interval series, included the standard deviation of all normal R-R intervals (SDNN, describing overall variation of $R-R$ intervals as estimate of overall $H R V$ ), and the root mean square of differences between successive R-R intervals (RMSSD, estimation of short-term HRV components, i.e, vagal tone $)^{4}$. With Poincaré analysis, SD1 represents the standard deviation of short-term $\mathrm{R}-\mathrm{R}$ variability as a result of parasympathetic efferent influences on the SA node, while SD2 denotes long-term or slow variability of heart rate, representing global variation in $\mathrm{HRV}^{4,9}$. Orthostatic stress (change from supine to upright) was used to examine the ANS response to stress. SPSS version 23 was used for statistical analyses. The results are presented as means and standard deviations. Spearman's correlations were calculated to determine if statistical significant correlations exist. $\mathrm{P}<0.05$ was considered statistical significant.

\section{Results}

All subjects worked long irregular hours in stressful jobs involving armed protection. Working hours were dictated by the demands of the work and varied from a minimum of $40 \mathrm{hrs}$ per week up to, occasionally, $80 \mathrm{hrs}$ per weekdepending on the nature of the deployment. Deployment could start in the early morning hours and/or spill over into night time. 
Table 1: Baseline characteristics of study population

\begin{tabular}{llll}
\hline Variable & Average \pm std & Minimum & Maximum \\
\hline Age (years) & $43.3 \pm 8.5$ & 28 & 57 \\
Weight $(\mathrm{kg})$ & $87.6 \pm 15.3$ & 56.8 & 123.0 \\
Height $(\mathrm{cm})$ & $172.0 \pm 7.3$ & 152.5 & 179.0 \\
BMI $\left(\mathrm{kg} / \mathrm{m}^{2}\right)$ & $29.6 \pm 4.9$ & 22.9 & 39.3 \\
Waist circumference $(\mathrm{cm})$ & $98.6 \pm 13.1$ & 73.5 & 132.5 \\
Hip circumference $(\mathrm{cm})$ & $100.5 \pm 9.3$ & 80.5 & 116.5 \\
Waist- to-hip ratio & $0.98 \pm 0.08$ & 0.86 & 1.14 \\
HDL-C (mmol/L) & $1.1 \pm 0.3$ & 0.6 & 2.2 \\
LDL-C (mmol/L) & $2.5 \pm 0.7$ & 1.1 & 4.0 \\
TotChol (mmol/L) & $4.4 \pm 0.9$ & 2.4 & 6.1 \\
HbA1c $(\%)$ & $5.7 \pm 0.4$ & 5.0 & 6.5 \\
HbA1c (mmol/mol) & $37.7 \pm 5.0$ & 31.0 & 48.0 \\
Glucose $(\mathrm{mmol} / \mathrm{L})$ & $5.7 \pm 0.8$ & 4.4 & 7.7 \\
CRP (mg/L) & $4.4 \pm 3.2$ & 1.0 & 11.0 \\
Cortisol (nmol/24h) & $61.0 \pm 34.6$ & 11.7 & 143.2 \\
Aldosterone (pmol/L) & $200.2 \pm 141.9$ & 39.0 & 479.0 \\
Albumin (g/L) & $41.4 \pm 2.4$ & 37.0 & 46.0 \\
Allostatic load & $4.4 \pm 2.4$ & 1 & 9 \\
\hline
\end{tabular}

$\mathrm{BMI}=$ body mass index; HDL-C=high density lipoprotein cholesterol; LDL-C=low density lipoprotein cholesterol; TotChol=total cholesterol; HbAlc=glycosylated haemoglobin.

The baseline characteristics of the group can be seen in Table 1. The mean allostatic load for the group was 4.4 \pm 2.4 . The subject with the lowest health risk had an allostatic load of 1 , while the subject with the highest health risk had an allostatic load of 9. The BMI for the group was $29.6 \pm 4.9 \mathrm{~kg} / \mathrm{m}^{2}$, indicating that almost half of the subjects were above the ideal BMI.

Associations between allostatic load and HRV indicator values (Total HRV and vagal activity dependent HRV) can be seen in Table 2. The results for both Total HRV and vagal HRV are given as obtained by frequency domain analysis based on Fast Fourier Transformations (FFT), by frequency domain analysis based on autoregression (AR), by time-domain analysis and by Poincaré analyses. Statistical significant inverse correlations were found between allostatic load and total HRV with results obtained by all HRV analytical techniques for baseline recordings $(\mathrm{Ph} 0)$, in response to the orthostatic challenge (Ph1), as well as for the first and second periods of standing (Ph2, $\mathrm{Ph} 3$ ). Inverse correlations were similarly found between allostatic load and vagal measures of HRV, as obtained by frequency domain analyses, for $\mathrm{Ph} 0, \mathrm{Ph} 1$, and $\mathrm{Ph} 2$ and between allostatic load and vagal measures of HRV as obtained by time domain and Poincaré analyses, for $\mathrm{Ph} 0$ and $\mathrm{Ph} 3$. 
Table 2: Associations between allostatic load and heart rate variability indicators based on frequency domain, time domain and Poincaré analyses $(n=27)$

\begin{tabular}{|c|c|c|c|c|c|c|c|c|}
\hline & \multirow{2}{*}{\multicolumn{2}{|c|}{$\begin{array}{l}\text { Frequency domain (FFT) } \\
\text { Total HRV }\end{array}$}} & \multirow{2}{*}{\multicolumn{2}{|c|}{$\begin{array}{l}\text { Frequency domain (AR) } \\
\text { Total HRV }\end{array}$}} & \multirow{2}{*}{\multicolumn{2}{|c|}{$\begin{array}{l}\text { Time domain } \\
\text { Total HRV (SDNN) }\end{array}$}} & \multirow{2}{*}{\multicolumn{2}{|c|}{$\begin{array}{l}\text { Poincaré } \\
\text { Total HRV (SD2) }\end{array}$}} \\
\hline & & & & & & & & \\
\hline & $\mathrm{r}$ & $\mathrm{p}$ & $\mathrm{r}$ & $\mathrm{p}$ & $\mathrm{r}$ & $\mathrm{p}$ & $\mathrm{r}$ & $\mathrm{p}$ \\
\hline $\mathrm{Ph} 0$ & -0.670 & $<0.001$ & -0.667 & $<0.001$ & -0.658 & $<0.001$ & -0.562 & 0.002 \\
\hline $\mathrm{Ph} 1$ & -0.569 & 0.002 & -0.565 & 0.003 & -0.530 & 0.004 & -0.451 & 0.018 \\
\hline $\mathrm{Ph} 2$ & -0.558 & 0.002 & -0.551 & 0.003 & -0.561 & 0.002 & -0.423 & 0.028 \\
\hline \multirow[t]{3}{*}{$\mathrm{Ph} 3$} & -0.469 & 0.014 & -0.497 & 0.008 & -0.484 & 0.010 & -0.499 & 0.008 \\
\hline & \multicolumn{2}{|c|}{ Vagal measure (HF) } & \multicolumn{2}{|c|}{ Vagal measure (HF) } & \multicolumn{2}{|c|}{ Vagal measure (RMSSD) } & \multicolumn{2}{|c|}{ Vagal measure (SD1) } \\
\hline & $\mathrm{r}$ & $\mathrm{p}$ & $\mathrm{r}$ & $\mathrm{p}$ & $\mathrm{r}$ & $\mathrm{p}$ & $\mathrm{r}$ & $\mathrm{p}$ \\
\hline $\mathrm{Ph} 0$ & -0.592 & 0.001 & -0.584 & 0.001 & -0.594 & 0.001 & -0.590 & 0.001 \\
\hline $\mathrm{Ph} 1$ & -0.458 & 0.016 & -0.368 & 0.059 & -0.323 & 0.100 & -0.323 & 0.10 \\
\hline $\mathrm{Ph} 2$ & -0.443 & 0.021 & -0.412 & 0.033 & -0.411 & 0.033 & -0.407 & 0.035 \\
\hline $\mathrm{Ph} 3$ & -0.327 & 0.096 & -0.369 & 0.058 & -0.296 & 0.134 & -0.303 & 0.124 \\
\hline
\end{tabular}

$\mathrm{FFT}=$ Fast Fourier Transformation; $\mathrm{AR}=$ Autoregression; HRV = heart rate variability; $\mathrm{Ph} 0=\mathrm{Phase} 0$ recording

( 5 min baseline, supine); $\mathrm{Ph} 1=$ Phase 1 recording (getting up from supine to standing and remain standing for $5 \mathrm{~min}$ ); $\mathrm{Ph} 2=\mathrm{Phase} 2$ recording ( 5 min standing, following $\mathrm{Ph} 1$ ); $\mathrm{Ph} 3=$ Phase 3 recording (5min standing, following $\mathrm{Ph} 2$ ); $\mathrm{HF}=$ High frequency band, represents predominantly vagal activity; SDNN = Standard deviation of all normal RR intervals, estimate of overall HRV; RMSSD = Root mean square of the differences between successive RR intervals, estimation of vagal power; SD1 = Standard deviation of the immediate or short-term RR variability as a result of vagal influence; SD2 = Indicator of long-term or slow variability of heart rate, representative of the global HRV.

Positive correlations were found between allostatic load and age $(r=0.625, \mathrm{p}=0.001)$ and between allostatic load and standing heart rate $(\mathrm{Ph} 2: \mathrm{r}=0.404, \mathrm{p}=0.037)$. Negative correlations were found between allostatic load and the heart rate response to orthostatic stress $(\% \Delta \mathrm{HR} \mathrm{Ph} 0$ to Ph1: $\mathrm{r}=-0.744, \mathrm{p}=0.001 ; \% \Delta \mathrm{HR} \mathrm{Ph} 0$ to $\mathrm{Ph} 2 \mathrm{:} \mathrm{r}=-0.679$, $\mathrm{p}=0.001)$. Negative correlations, at significance levels ranging from $\mathrm{p}<0.05$ to $\mathrm{p}<0.001$, were found between age and total HRV, and between age and vagal-mediated HRV indicators, for all HRV analytical methods used.

Increases in pro-inflammatory activity, as reflected by CRP levels, were found with a decline in total HRV and vagal measures of HRV for all four phases, i.e, the supine phase $(\mathrm{Ph} 0)$, rising from supine to standing $(\mathrm{Ph} 1)$ and the two 5 minute phases during which subjects remained stationary in the standing position ( $\mathrm{Ph} 2$ and $\mathrm{Ph} 3)$. For example, results obtained by frequency domain analysis based on FFT showed negative correlations between CRP and total HRV for Ph0 ( $\mathrm{r}=-0.576, \mathrm{p}=0.002)$, for $\mathrm{Ph} 1$ $(\mathrm{r}=-0.532, \mathrm{p}=0.004)$, for $\mathrm{Ph} 2(\mathrm{r}=-0.548,0.003)$ and for Ph3 ( $\mathrm{r}=-0.525, \mathrm{p}=0.005)$, as well as negative correlations between CRP and HF for $\mathrm{Ph0}(\mathrm{r}=-0.467, \mathrm{p}=0.014)$, for Ph1 $(\mathrm{r}=-0.538, \mathrm{p}=0.004)$, for Ph2 $(\mathrm{r}=-0.549, \mathrm{p}=0.003)$ and for Ph3 ( $\mathrm{r}=-0.468, \mathrm{p}=0.014)$.

\section{Discussion}

Allostatic load scores and HRV have repeatedly been used African Health Sciences Vol 17 Issue 2, June, 2017 as indicators of stress-related health risks ${ }^{1,2,3,10}$. HRV declines and allostatic load increases with prolonged psychosocial stress ${ }^{1,2,3,10}$. The first question asked was whether health risk assessment by allostatic load and that by HRV give comparable results. The second question was whether it is meaningful to include selected HRV indicators as indices of allostatic load, and if so, which indicators.

Results of the present study confirmed previous reports $^{11,12}$ that allostatic load increases with age and HRV decreases with age. As seen in Table 1, decreases in total HRV correlated at a statistical significant level with increases in allostatic loads for recordings in the supine position ( $\mathrm{Ph} 0$ ), for the 5 minute period that subjects changed from supine to upright (Ph1), as well as for the two 5 minute periods of standing (Ph2, Ph3). With minor differences in significance levels, results on total HRV were in agreement, whether obtained by frequency domain, time domain (SDNN) or Poincaré analysis (SD2). Correlations with the highest levels of significance were seen for the baseline (supine) recordings (Table 1).

These inverse associations between allostatic load and total HRV are in line with the fact that allostatic load increases and HRV decreases with stress and stress-related disease. High allostatic loads have, for instance, been associated with psychosocial stress, stress related mental 
and physical disorders and with increased risks for allcause mortality ${ }^{1,3,13}$, while low HRV is associated with increased psychosocial stress, several physical and psychological disorders and with increased risk for all-cause mortality ${ }^{4,5,10}$. The results thus indicate a good inverse association between total HRV and allostatic load scores.

The next association investigated was that between allostatic load and vagal activity as reflected by the vagal-mediated HRV indicators HF, RMSSD and SD1. In the present study (Table 2) significant negative correlations were seen between allostatic load and vagal-mediated HRV for recordings in the supine $(\mathrm{Ph} 0)$ and in the upright $(\mathrm{Ph} 2)$ position. It seems feasible to assume the lack of a correlation between allostatic load and vagal tone during the period of rising from the supine to upright (Ph1) to be the result of the vagal withdrawal known to occur as part of the ANS orthostatic response and which, in turn, depends on vagal flexibility. Vagal flexibility in response to a challenge/stressor is generally seen as a measure of a positive ANS status, but the relationship may not necessarily be linear ${ }^{13}$. In the present study, correlations between allostatic loads and the vagal responses to orthostatic stress were not of statistical significance.

The significant negative correlations between allostatic load scores and the vagal indicators HF, RMSSD and SD1 suggest a good correspondence between health risk assessments based on allostatic load and that based on vagal measures of HRV. In view of the regulatory role of the vagus nerve in cardiovascular and metabolic function, as well as the decrease in vagal tone previously reported with cardiovascular and metabolic disturbances ${ }^{14}$, this does not come as a surprise.

In addressing the question of whether HRV indicators should be included in assessments of allostatic load, it is necessary to first look at the original measurements on which the calculation of allostatic load was based. In that set of ten variables, indications of ANS status (as primary mediator) was derived from urinary noradrenalin and adrenalin excretion as indices of sympathetic nervous systems activity and indirectly by indices of cardiovascular function (secondary outcomes) ${ }^{3}$. Due to methodological issues and ostensibly the fact that noradrenalin and adrenalin excretion reflects adrenal medullary function, rather than that of the sympathetic nervous system per se, urine noradrenalin and adrenalin are in reality seldom measured $^{1}$. This not only contributes to heterogeneity in the type and number of markers used, but also leaves a gap with regard to a primary mediator that reflects ANS function. In view of the latter, and the fact that HRV analysis provides a recognised window into ANS function, HRV appears to be ideal for inclusion, and probably as replacement for urine adrenalin and noradrenalin, in the calculation of allostatic load.

In deciding which HRV indicators should be included as allostatic indices there can be no argument about the inclusion of total HRV as it has repeatedly been shown as reliable indicator of well-being. We are thus faced with the conundrum relating to the two branches of the ANS. A strong case exists for inclusion of vagal, rather than sympathetic, measures of HRV. The first issue is the strong reservations about the validity of HRV-derived information on sympathetic nervous system activity ${ }^{8}$.

In contrast to sympathetic indicators, it is generally accepted that vagal measures of HRV are reliable and fair indications exist for their temporal stability, if only over shorter intervals ${ }^{15}$. Another reason for the inclusion of vagal indicators is to be found in the pervasive role played by the vagus nerve in homeostatic processes and the fact that cardiovascular and metabolic disturbances are marked by a decrease in vagal tone ${ }^{14,16}$. The inverse correlations found in the present study between vagal measures of HRV and allostatic load scores are, in fact, in support of the concept of vagal activity as major regulator of allostatic systems such as the HPA-axis and pro-inflammatory immune activity ${ }^{17}$.

In line with the above, and the previously reported link between impairment in vagal nerve activity and dysregulation of inflammatory processes ${ }^{18}$, one would have expected indications of an increase in pro-inflammatory activity with a decline in vagal tone. This was indeed the case as suggested by the inverse associations found between the levels of the pro-inflammatory marker, CRP, and all vagal measures of HRV. Of interest, is the fact that pro-inflammatory cytokines are, in turn, also involved in the regulation of a host of physical and psychological activities in the body and have been described in association with stress and lifestyle associated diseases, including metabolic and cardiovascular disorders and even as a major factor 
in the multiple system deterioration of pathological aging - a condition popularly referred to as inflammaging ${ }^{19,20}$. The correlation between vagal and inflammatory activity, found in the present study, thus further supports the inclusion of a vagal measure of HRV into the assessment of allostatic load.

A number of studies have previously incorporated indicators of HRV into allostatic load assessments ${ }^{1}$. Support for our view of including a vagal measure of HRV into allostatic load assessment comes from a recent paper where allostatic load assessments on 12,477 industrial workers was based on a set of five variables, including RMSSD as measure of vagal activity ${ }^{21}$. In the latter study, HRV tachograms were recorded over an unspecified number of day time hours while subjects were involved in normal work and leisure activities. Exact details about the length of recordings and analytical technique are unfortunately not given.

The main problem with the introduction of HRV values as part of allostatic load is the inconsistency with regard to the choice of HRV indicators, the choice of analytical techniques and the conditions and time periods under which the tachogram recordings are made. It is our contention that HRV does indeed have a place in the assessment of allostatic load and that both total HRV and vagal measures of HRV would give reliable results, but that the latter would be the better choice because of the pervasive role played by vagal activity in cardiovascular, metabolic and immune regulatory processes. In addition, for the sake of consistency, recordings should be done under controlled conditions in a specific body position, and consensus should be reached on which analytical technique is best suited. In our view, short term baseline (supine) recordings, under controlled conditions, analysed by either frequency or time domain techniques would minimize the introduction of methodology-related variations.

Another possibility that should perhaps be investigated for inclusion into allostatic load scores is the heart rate response and recovery in reaction to orthostatic stress as an alternative to HRV responses to orthostatic stress. The heart rate response to orthostatic stress is largely a function of ANS flexibility and fairly easy to assess. It will, however, require both upper and lower cut-off points for the final analysis. In the present study, highly significant negative correlations were found between the heart rate responses to the orthostatic stressor and allostatic load scores.

\section{Conclusion}

Total HRV and vagal indicators of HRV give results comparable to that of allostatic load scores when used as health risk indicators. Total HRV and vagal, rather than sympathetic measures of HRV, are indicated for inclusion into allostatic load assessments. The inclusion of heart rate responses to orthostatic stress, as indices of ANS function, should be further investigated. The results of this study are in line with the concept of vagal tone as a regulator of allostatic systems.

\section{Limitations of the study}

Due to the small group of subjects, this study could perhaps be considered preliminary and should be followed up on a much larger group.

\section{References}

1. Mauss D, Li J, Schmidt B, Angerer P, Jarczok MN. Measuring allostatic load in the workforce: A systematic review. Industrial Health 2015; 53(1): 5-20.

2. McEwen BS, Stellar E. Stress and the individual: Mechanisms leading to disease. Archives of Internal Medicine 1993; 153(18): 2093-2101.

3. Seeman TE, Singer BH, Rowe JW, Horwitz RI, McEwen BS. Price of adaptation - allostatic load and its health consequences: MacArthur studies of successful aging. Archives of Internal Medicine 1997; 157(19): 2259-2268.

4. Task Force of The European Society of Cardiology and The North American Society of Pacing and Electrophysiology. Heart rate variability. Standards of measurement, physiological interpretation, and clinical use. European Heart Journal 1996; 17: 354-381.

5. Kemp AH, Quintana S. The relationship between mental and physical health: Insights from the study of heart rate variability. International Journal of Psychophysiology 2013; 89(3): 288-296.

6. Schroeder EB, Chambless LE, Liao D, et al. Diabetes, glucose, insulin, and heart rate variability: The atherosclerosis risk in communities (ARIC) study. Diabetes Care 2005; 28(3): 668-674. 
7. Tarvainen MP, Ranta-aho PO, Karjalainen PA. An advanced detrending method with application to HRV analysis. IEEE Transactions on Biomedical Engineering 2002; 49(2): 172-175.

8. Reyes del Paso GA, Langewitz W, Mulder LJM, van Roon A, Duschek S. The utility of low frequency heart rate variability as an index of sympathetic cardiac tone: $A$ review with emphasis on a reanalysis of previous studies. Psychophysiology 2013; 50(5): 477-487.

9. Tarvainen MP, Niskanen JP, Lipponen JA, Ranta-Aho PO, Karjalainen PA. Kubios HRV - A software for advanced heart rate variability analysis. $4^{\text {th }}$ European Conference of the International Federation for Medical and Biological Engineering, ECIFMBE 2008, Antwerp. , 22 1022-1025.

10. Jarczok MN, Jarczok M, Mauss D, Koenig J, Li J, Herr RM, Thayer JF. Autonomic nervous system activity and workplace stressors--a systematic review. Neuroscience and Biobehavioral Reviews 2013; 37(8): 1810-1823.

11. Crimmins EM, Johnston M, Hayward M, Seeman T. Age differences in allostatic load: An index of physiological dysregulation. Experimental Gerontology 2003; 38(7): 731-734.

12. Zhang J. Effect of age and sex on heart rate variability in healthy subjects. Journal of Manipulative and Physiological Therapentics 2007; 30(5): 374-379.

13. Kogan A, Gruber J, Shallcross AJ, Ford BQ, Mauss IB. Too much of a good thing? Cardiac vagal tone's non- linear relationship with well-being. Emotion 2013; 13(4): 599-604.

14. Thayer JF, Lane RD. The role of vagal function in the risk for cardiovascular disease and mortality. Biological Psychology 2007; 74(2): 224-242.

15. Bertsch K, Hagemann D, Naumann E, Schächinger $\mathrm{H}$, Schulz A. Stability of heart rate variability indices reflecting parasympathetic activity. Psychophysiology 2012; 49(5): 672-682.

16. Thayer JF, Yamamoto SS, Brosschot JF. The relationship of autonomic imbalance, heart rate variability and cardiovascular disease risk factors. International Journal of Cardiology 2010; 141(2): 122-131.

17. Thayer JF, Sternberg E. Beyond Heart Rate Variability. Annals of the New York Academy of Sciences 2006; 1088: 361-372.

18. Pavlov VA, Tracey KJ. The vagus nerve and the inflammatory reflex - linking immunity and metabolism. Nature Reviews Endocrinology 2012; 8(12): 743-754.

19. Kao PC, Shiesh SC, Wu TJ. Review: Serum C-reactive protein as a marker for wellness assessment. Annals of Clinical and Laboratory Science 2006; 36(2): 163-169).

20. Michaud M, Balardy L, Moulis G, et al.. Proinflammatory cytokines, aging, and age-related diseases. Journal of the American Medical Directors Association 2013; 14(12): 877-882.

21. Mauss D, Jarczokc MN, Fischera JE. The streamlined Allostatic Load Index: a replication of study results. Stress 2016;19(6):553-558. 